\title{
Recognition for lateral faces using Neural Networks
}

\author{
Dr.M.Aruna Safali ${ }^{\mathrm{a}}$, Rajitha Laxmi.Ch ${ }^{\mathrm{b}}$, Y.Lavanya ${ }^{\mathrm{c}}$, Bhagyasri Pavuluri ${ }^{\mathrm{d}}$ \\ ${ }^{a}$ Assoc.Professor, Dept of IT, NRI Institute Of Technology \\ ${ }^{\mathrm{b}}$ Asst.Professor, Dept of ECE, RamaChandra College Of Engineering \\ ${ }^{c}$ Assoc.Professor, Dept of ECE, RamaChandra College Of Engineering \\ ${ }^{\mathrm{d}}$ Asst.Professor, Dept of ECE, RamaChandra College Of Engineering
}

Article History: Received: 10 November 2020; Revised 12 January 2021 Accepted: 27 January 2021; Published online: 5 April 2021

\begin{abstract}
Face recognition is most difficult and complicated technique. Recognition of lateral faces is very difficult compare with normal face recognition. Pattern recognition is mostly used in this system to recognise the lateral face patterns (LFP). Neural network is used to find the patterns and lateral face recognition can be done by this technique. After the many researches face recognition becomes difficulty for the various techniques based on their parameters. In this paper, the amalgamative lateral face recognition(ALFR) which is merged with machine learning and neural network features can be done by using synthetic dataset consists of 200 lateral faces. Performance shows the improved results of proposed technique.
\end{abstract}

Keywords: lateral face patterns (LFP), neural networks (NN), face recognition.

\section{Introduction}

Pattern recognition (PR) is a cutting edge machine learning issue with various applications in a vast field, including lateral face recognition (LFR), Character recognition (CR), Speech recognition (SR). The field of example acknowledgment is still especially in it is outset, in spite of the fact that as of late a portion of the boundaries that hampered such mechanized LFR has been lifted because of advances in PC equipment giving machines prepared to do the quicker and progressively complex calculation. FR is the most tedious task for the human brain. It is ordinarily utilized in applications, for example, human-machine interfaces and programmed access control frameworks. FR includes contrasting a picture and a database of put away faces so as to distinguish the person in that information picture. The related errand of face discovery has direct pertinence to confront acknowledgment since pictures must be broken down and faces distinguished before they can be perceived. Identifying faces in a picture can likewise center the computational assets of the face acknowledgment framework, streamlining the frameworks speed and execution. Face identification includes isolating picture windows into two classes; one containing faces (targets), and one containing the foundation (clutter). It is troublesome in light of the fact that despite the fact that shared characteristics exist between faces, they can differ significantly as far as age, skin shading and expression on faces.

LFR is an intriguing and effective use of Pattern acknowledgment and picture investigation. Facial pictures are basic for savvy vision-based human-PC association. Face preparing depends on the way that the data about a client's personality can be removed from the pictures and the PCs can act likewise. Face recognition has numerous applications, extending from diversion, Information security, and Biometrics [1]. Various strategies have been proposed to identify faces in a solitary picture. To construct completely mechanized frameworks, strong and effective face identification calculations are required. The face is identified once an individual's face comes into a view [2]. When a face is recognized, the face locale is edited from the picture to be utilized as "Test" into the information to check for potential matches. The face picture is preprocessed for variables, for example, picture size and enlightenment and to identify specific highlights. The information from the picture is then coordinated against the learning. The coordinating calculation will create a likeness measure for the match of the test face into the information.

An Amalgamative face recognition (AFR) strategy where nearby highlights are given as the contribution to the neural system. To start with, the face locale is separated from the picture by applying different pre-preparing exercises. The technique for finding the face district is known as face confinement. The neighborhood highlights, for example, eyes and mouth are removed from the face district. The separation between the eyeballs and the separation between the mouth endpoints are determined to utilize the distance computation algorithm. At that point the separation esteems between the left eye and the left mouth endpoint, the correct eye and the correct 
mouth endpoint, the left eye and the correct mouth endpoint, the correct eye, and the left mouth endpoint are determined. These qualities are given as contributions to the neural system.

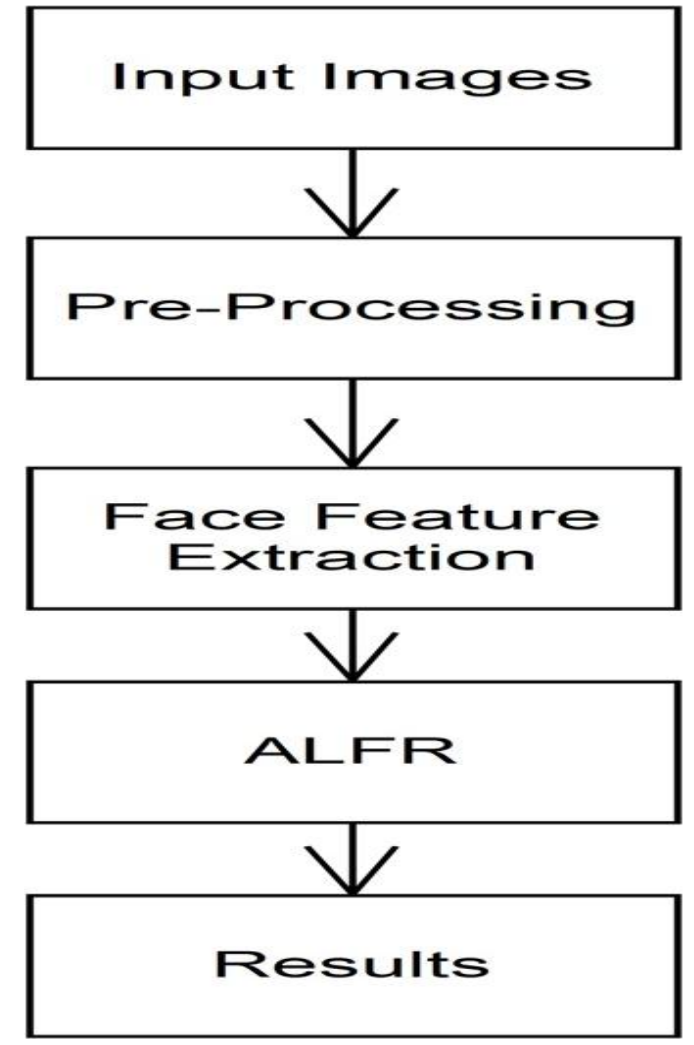

Figure: 1.1 System Architecture

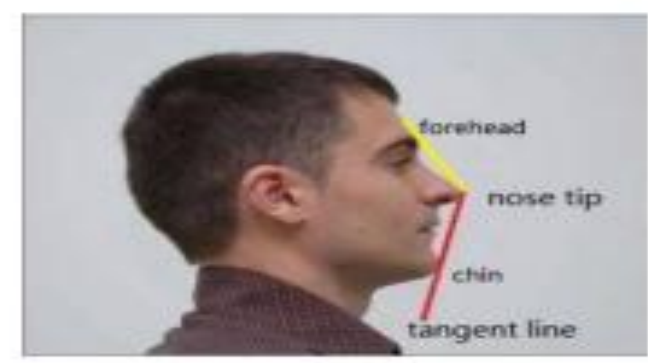

Figure: 2.1 Sample Lateral Face

\section{Methodologies}

In this chapter, various methodologies are discussed.

\section{Feature Based Lateral Face Recognition (FBLFR)}

The FBLFR method performs on human face. Input image can be in different face orientation where transformation of feature space is learned and applied on face for feature extraction. Objective is human's various face features like left eye, right eye, nose, mouth are to be extracted. Viola - Jones Skin detection method is best for feature extraction. In multiview face recognition as shown in Figure 3 and 4, face image pass as an input then local feature of face to be extracted. In result it develops mirror image of any best side of human face as 2D mug shown in Figure 3.1. 


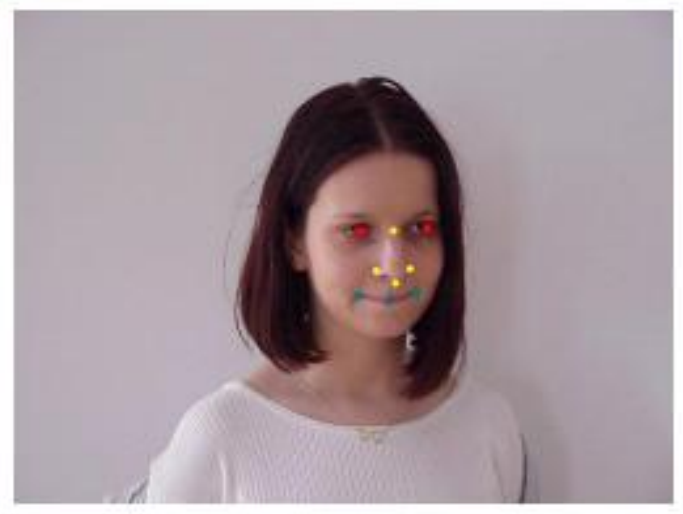

Figure: 3.1 Sample Lateral Faces

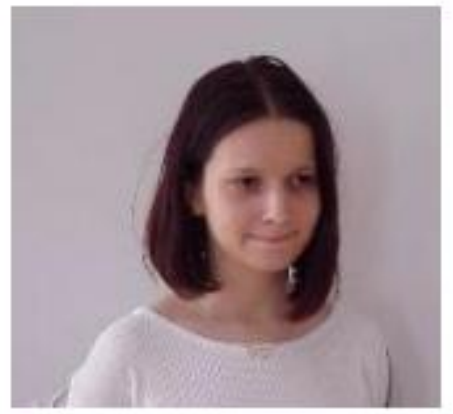

Figure: 3.2 Sample Lateral Faces

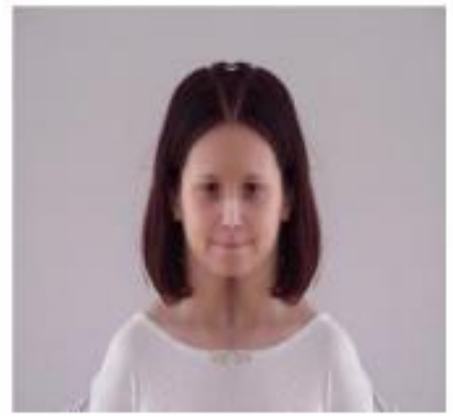

Figure: 3.3 Sample Lateral Faces

\subsection{ROI Face Detection \& Alignment}

It involves the only region of the face from datasets and target sample. These pictures can have human body segment like the neck, bind, fabric, top or whatever other things that aren't required for acknowledgment. Utilizing viola-jones face identification system creators remove just face area. Viola-jones face identification system has three indispensable advances: I) include extraction, ii) boosting iii) multi-scale discovery. Distinguished face district may have a minor cross face as of typical human behavior. It conceals any hint of failure district as an element vector for further examination.

\subsection{Face Features Vector Generation}

Target images may have other background objects too, so using viola-jones face detection technique proposed method extract available faces from target image scene. At the end of this phase all faces are extracted and store it properly for future verification operation. In normal environment human face may not be straight always, so in recognition method position of face feature landmark is changed, and as a result it gives less matching value so face recognition might be unsuccessful. For solution of this issue, we can rotate whole face by calculating distance from $\mathrm{X}$ and $\mathrm{Y}$ axis of both eyes.

$$
\begin{aligned}
& \mathrm{y}=\text { left eye from } \mathrm{Y} \text { axis }- \text { right eye from } \mathrm{Y} \text { axis } \\
& \mathrm{x}=\text { left eye from } \mathrm{X} \text { axis }- \text { right eye from } \mathrm{X} \text { axis }
\end{aligned}
$$


Rotation angle $=\arctan (\mathrm{y} / \mathrm{x})$

Using above equation we have rotate whole face to prepare it straight then send for further steps. End of this step all faces are extracted and store it in form of feature vector for feature extraction.

\subsection{Integrated Deep Model for Face Detection and Landmark Localization from "In the Wild" Images}

In recent years the face detection and landmark localization are two main factors in facial analysis applications. Many of the issues are solved to detect the face recognition which increases the precision of face detection [20]. This reference proposed the novel method the Integrated Deep Model (IDM) and adopted the two traditional deep learning techniques such as Faster R-CNN and a stacked hourglass which improves the face detection precision and accurate landmark localization. The optimization function is integrated with the proposed system which increases the accuracy and reduces the false positive rate which is 63\%. The IDM technique uses the Annotated Faces In-The-Wild, Annotated Facial Landmarks in The Wild and Face Detection Dataset and Benchmark face detection test sets and shows a high level of recall and precision when compared with various existing methods. The dataset used in this system is $300-\mathrm{W}$ test sets which are focused on localization accuracy with original bounding boxes. The increase with our proposed system is $0.005 \%$ maximum with facial landmarks which border the face.

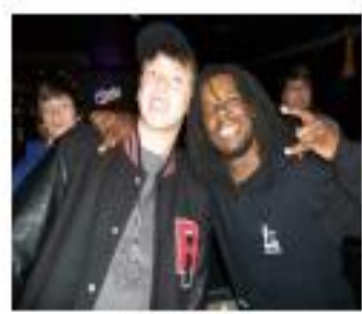

Input imsge

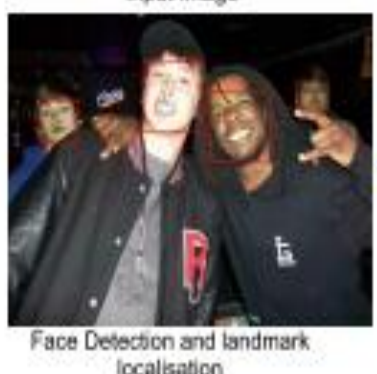

localisation

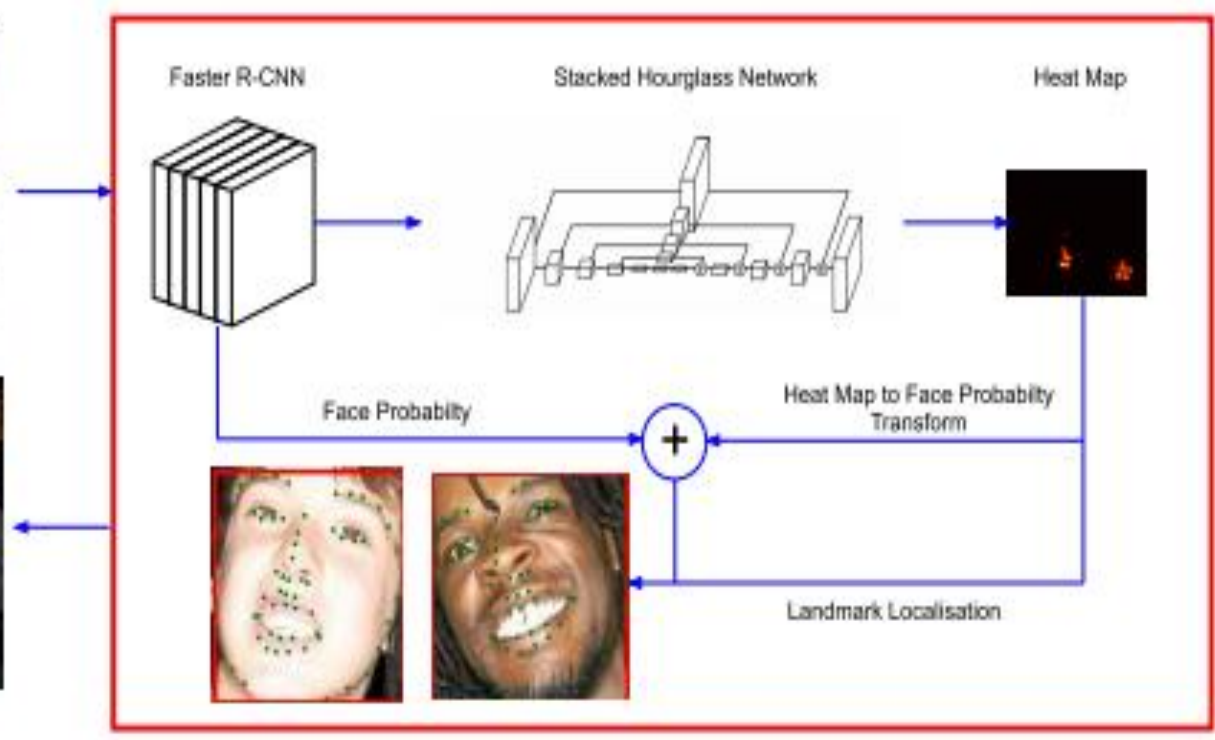

Figure: 3.6 Steps to process the IDM

\section{Deep Learning Face Attributes in the Wild}

Face attributes prediction is the most complicated issue to find the various complex face variations. In this system, they have shown the two categories such as LNet and ANet are the finely trimmed combined with tags and these are previously trained. For the face localization, LNet is the pre-trained traditional massive item and the other ANet is used to predict the attributes [21]. The proposed system provides not only accuracy but also the facts based on face learning. This will increase the face localization (LNet) and attribute prediction (ANet) with different pre-training techniques. The fine-tuned filters are used to get the image-level attribute tags and reply to the maps over all the images. This will also explain the high-level hidden neurons of ANet automatically which finds the semantic items after training with massive face findings.

\begin{tabular}{|l|l|l|l|}
\hline Method & Dataset & Description & Result \\
\hline Distribution Based & MIT test set & $\begin{array}{l}\text { Two sets of high and low } \\
\text { resolution gray scale } \\
\text { images with multiple } \\
\text { faces }\end{array}$ & $81.9 \%$ \\
\hline Neural Network & CMU test set & 130 gray scale images & $90.3 \%$ \\
\hline Naive Bayes & CMU profile face test set & 208 gray scale images & $93.2 \%$ \\
\hline $\begin{array}{l}\text { Kullback relative } \\
\text { Information }\end{array}$ & Kodak dataset & $\begin{array}{l}\text { Faces of multiple size, } \\
\text { pose and under varying. }\end{array}$ & $98.0 \%$ \\
\hline
\end{tabular}




\section{Experiments}

This is the synthetic dataset which consists of various lateral faces that can be used for training and then the input is given to system for face detection of lateral faces. These experiments are done by using the java programming language. There are 3 parameters are shown in the bases of performance such as sensitivity, specificity and accuracy.

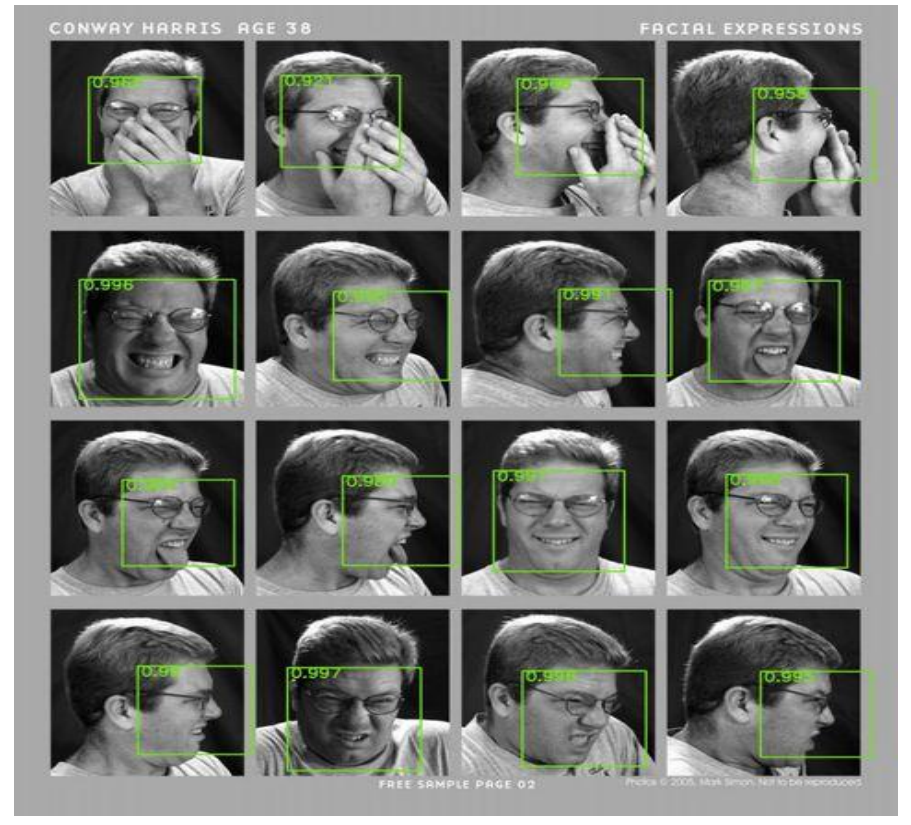

Figure: 6 Dataset image
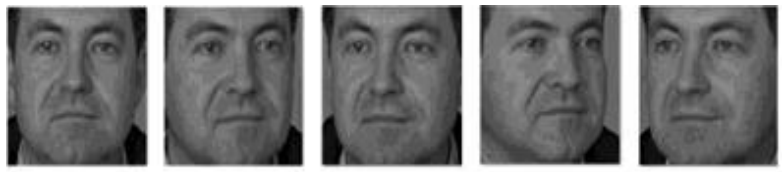

(a) Ground truth for Subject 00019
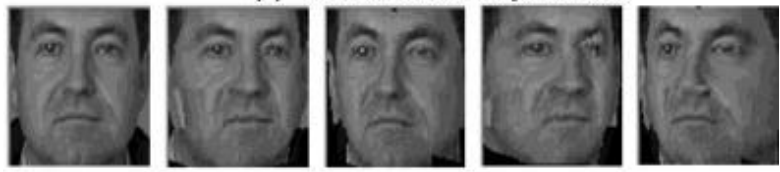

(b) Virtual poses for Subject 00019
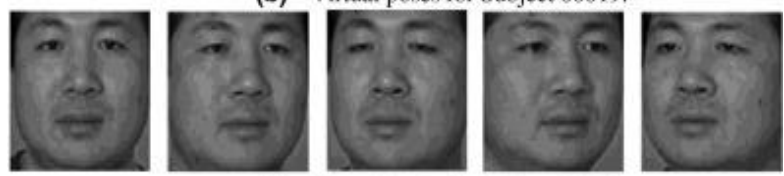

(c) Ground truth for Subject 00029
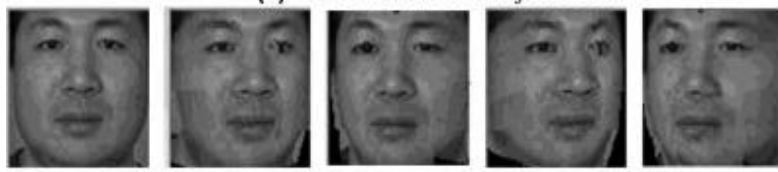

(d) Virtual poses for Subject 00029

Figure: 7 Dataset image

\section{Performance Evolution}

By utilizing the performance measures namely False Positive Rate, False Negative Rate, Sensitivity, Specificity and Accuracy, the performance of the system is estimated. The basic count values such as True Positive (TP), True Negative (TN), False Positive (FP) and False Negative (FN) are used by these measures.

\section{False Positive Rate (FPR)}

The percentage of cases where an image was classified to normal images, but in fact it did not. 


$$
F P R=\frac{F P}{F P+T N}
$$

\section{False Negative Rate (FNR)}

The percentage of cases where an image was classified to abnormal images, but in fact it did.

$$
F N R=\frac{F N}{F N+T N}
$$

\section{Sensitivity}

The proportion of actual positives which are correctly identified is the measure of the sensitivity. It relates to the ability of the test to identify positive results.

$$
\text { Sesitivity }=\frac{\text { No. of TP }}{\text { No. of TP +No.of TN }}
$$

The proportion of negatives which are correctly identified is the measure of the specificity. It relates to the ability of the test to identify negative results.

$$
\text { Specificity }=\frac{\text { No. of TN }}{\text { No.of TN }+ \text { No.of FP }}
$$

The following steps are utilized by the ALFR system

1.) Initialize the lateral face 0 images from the datasets.

2.) Pre-processing the samples.

3.) Training the samples.

4.) Start matching the sample images.

5.) Calculate the sensitivity

6.) Specificity and Accuracy.

7.) Show results

8.) Stop

\begin{tabular}{|l|l|l|l|}
\hline & Accuracy & Sensitivity & Specificity \\
\hline ALFR & $\mathbf{8 9 \%}$ & $\mathbf{8 6 \%}$ & $\mathbf{8 9 \%}$ \\
\hline
\end{tabular}

Table: 1 Performance of Integrated system in terms of Accuracy

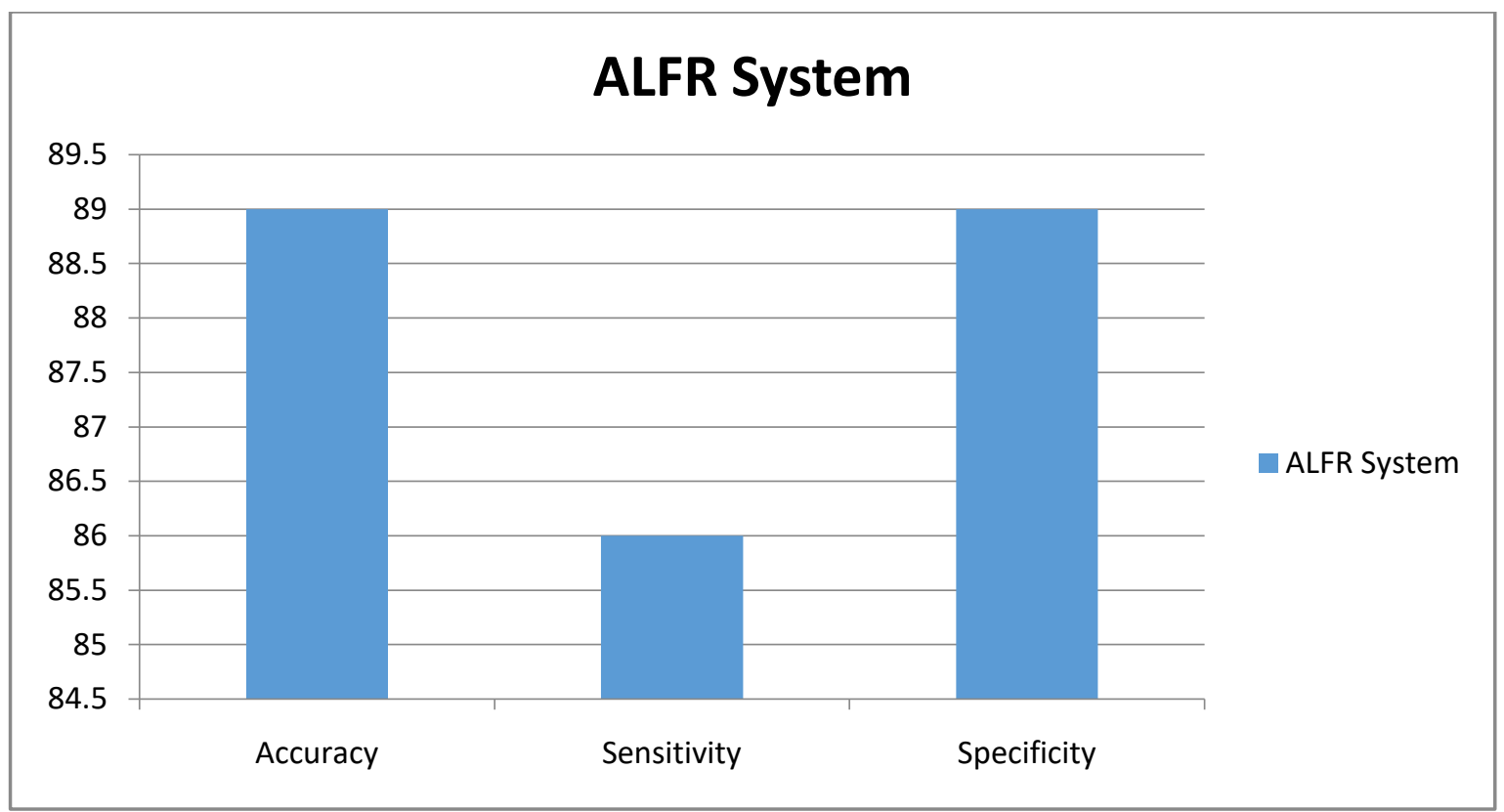

Figure: 11 Show the performance of Integrated System 


\section{Conclusion}

In this paper, the proposed system focuses on improving the accuracy, sensitivity and specificity for the lateral faces. It is very needed for every face recognition according to the lateral faces. Recognition of lateral faces is mostly difficult to get the accurate result. But the proposed system works according to the input lateral face image.

\section{References}

X. Zhang, Y. Gao "Face recognition across pose: A review" Pattern Recognition 42(11) (November 2009) 28762896

T. Ahonen, A. Hadid, M. Pietik"ainen. "Face recognition with local binary patterns" In Proc. of ECCV, 2004

B. Babenko, P. Doll'ar, Z. Tu, S. Belongie "Simultaneous learning and alignment: Multi-instance and multi-pose learning" In Workshop on Faces in 'Real-Life' Images: Detection, Alignment, and Recognition, 2008

A.F.Abate,M.Nappi,D.Riccio,G.Sabatino "2D and 3D face recognition: a survey" Pattern Recognition Lett.28(14)(2007) 1885- 1906

U. Kurmi, D. Agrawal. Study of Different Face Recognition Algorithms and Challenges. International Journal of Engineering Research (ISSN:2319-6890)(online),2347-5013(print) Volume No.3, Issue No.2, pp : 112-115 01 Feb. 2014

D.Jiang,Y.Hu,S.Yan,L.Zhang,H.Zhang,W.Gao, Efficient 3D reconstruction for face recognition, Pattern Recognition 38(6)(2005)787-798

A. Maghraby, M. Abdalla, "Hybrid Face Detection System using Combination of Viola - Jones Method and Skin Detection" International Journal of Computer Applications (0975 - 8887) Volume 71- No.6, May 2013

C.D.CastilloandD.W.Jacobs,Using stereo matching for 2-D face recognition across pose,in:ProceedingsoftheIEEEConferenceonCVPR,2007,pp.1-8

V.Blanz,T.Vetter,Facerecognition based on fitting a 3D morphable model, IEEE Trans.PatternAnal.Mach.Intell.25(9)(2003) 1063-1074

A.S.Georghiades,P.N.Belhumeur,D.J.Kriegman,Fromfewtomany: illumination cone models for face recognition undervariable lighting and pose, IEEE Trans.PatternAnal.Mach.Intell.23(6)(2001)643-660

K.W.Bowyer,C.Kyong,P.Flynn, A survey of approaches and challenges in 3D and multi-modal 3D + 2D face recognition , Comput.VisionImageUnderstanding 101 (1)(2006)1-15

Pantic, M., Valstar, M., Rademaker, R., Maat, L.: Web-based database for facial expression analysis. In: IEEE Int. Conf. on Multimedia and Expo, IEEE (2005) 317-321

V.Blanz,T.Vetter, Face recognition based on fittinga 3D morphablemodel, IEEE Trans.Pattern Anal.Mach.Intell.25(9) (2003)1063-1074

Aghajanian J. and Prince S., "Face Pose Estimation in Uncontrolled Environments," International Conference on Computer Vision, available at: http://www.bmva.org/bmvc/2009/ Papers/Paper256/Abstract256.pdf, last visited 2009

C. Moujahdi, S. Ghouzali. Inter-Communication Classification for Multi-View Face Recognition. The International Arab Journal of Information Technology, Vol. 11, No. 4, July 2014

Zhaoxiang Zhang, Chao Wang and Yunhong Wang, "Video-Based Face Recognition: State of the Art".

V. Kr"ueger and S. Zhou.,"Exemplar-based face recognition from video.”, In Proc. European Conf. on Computer Vision, volume 4, pp: 732-746.

Masi et al., "Learning Pose-Aware Models for Pose-Invariant Face Recognition in the Wild," in IEEE Transactions on Pattern Analysis and Machine Intelligence, vol. 41, no. 2, pp. 379-393, 1 Feb. 2019.

J. Zhao, J. Han and L. Shao, "Unconstrained Face Recognition Using a Set-to-Set Distance Measure on Deep Learned Features," in IEEE Transactions on Circuits and Systems for Video Technology, vol. 28, no. 10, pp. 2679-2689, Oct. 2018.

G. Storey, A. Bouridane and R. Jiang, "Integrated Deep Model for Face Detection and Landmark Localization From "In The Wild" Images," in IEEE Access, vol. 6, pp. 74442-74452, 2018.

Z. Liu, P. Luo, X. Wang and X. Tang, "Deep Learning Face Attributes in the Wild," 2015 IEEE International Conference on Computer Vision (ICCV), Santiago, 2015, pp. 3730-3738 